\title{
Clinical characteristics and pathogens of pediatric pyogenic liver abscess in Beijing Children's Hospital, China
}

\section{Yue Xie}

Beijing Children's hospital https://orcid.org/0000-0003-0115-1241

\section{Ling-yun Guo}

Beijing Children's hospital

Lin-lin Liu

Beijing Children's hospital

Bing Liu

Beijing Children's hospital

\section{Wen-ya Feng}

Beijing Children's hospital

Hui-li Hu

Beijing Children's hospital

Bing Hu

Beijing Children's hospital

\section{Xin Guo}

Beijing Children's hospital

Tian-ming Chen

Beijing Children's hospital

\section{Shao-ying Li}

Beijing Children's hospital

\section{Su-ying Qian}

Beijing Children's hospital

\section{Ming-Yan Hei}

Beijing Children's hospital

Hu-yong Zheng

Beijing Children's hospital

Gang Liu ( $\square$ liugangbch@sina.com )

https://orcid.org/0000-0001-8551-741X 
Keywords: Pyogenic liver abscess, Pathogen, Children

Posted Date: January 30th, 2020

DOI: https://doi.org/10.21203/rs.2.22233/v1

License: (c) (i) This work is licensed under a Creative Commons Attribution 4.0 International License. Read Full License 


\section{Abstract}

Background: Data on pyogenic liver abscess (PLA) of children in China have been limited.

Method: We retrospectively reviewed PLA cases from January 2017 to June 2019 at Beijing Children's Hospital. Clinical features and pathogens were analyzed.

Results: We diagnosed 15 PLA patients in our center. Median age of onset was 3.2 years and the median diagnostic time was 9 days. Four patients $(26.7 \%)$ had underlying diseases and seven patients $(46.7 \%)$ had multiple organ involvement. The sensitivity achieved $100 \%$ when ultrasound and contrast-enhanced CT scan were combined use to diagnose PLA. Two patients were defined pathogen through metagenomic next-generation sequencing (mNGS). Finally, eleven patients (73.3\%) were cured during follow-up time and four patients $(26.7 \%)$ dead. Compared to the survival group and the death group, the patients in death group were more likely to have multiple organ involved (100 vs. $27 \%, \mathrm{P}<0.05)$ and bilateral lobe occupied $(P<0.05)$.

Conclusions: PLA often occurs in patients with underlying disease. Multiple organs involved may lead to poor prognosis. mNGS can be a new method to detect pathogen.

\section{Background}

Pyogenic liver abscess (PLA) is considered as an uncommon infection whereas comprises the majority of hepatic abscess $(\mathrm{HA})^{[1]}$. It was associated with a high prevalence and significant mortality in the past years, with the incidence of 5 to 20 in 100,000 hospitalizations among adults ${ }^{[2]}$. However, according to the limited reports in children, the incidence of PLA is higher in developing countries. For instance, the morbidity was 1 in 140 admissions in Brazil ${ }^{[3]}, 78.9$ per 100,000 admissions in South India [4]. Nevertheless, the incidence of PLA is reported to be lower among children in developed countries, with 25 per 100,000 admissions in US ${ }^{[5]}$. And in Taiwan, was 8.9 to 20 per 100,000 admissions ${ }^{[6,7]}$.

Overall, Staphylococcus aureus (S.aureus) is frequently isolated from PLA in children. Escherichia coli (E. coli), Klebsiella pneumonia (K.pneumonia) and Enterobacter are also the common pathogens ${ }^{[3,8,9]}$. In recent years, investigations found the prevalence of K.pneumonia infection increased worldwide. Similarly, upward tendency was shown in China ${ }^{[8]}$. Patients with underlying diseases are tending to get PLA. Diabetic mellitus (DM), cirrhosis, immune-compromised state are the well-known predisposing factors ${ }^{[10]}$.

But so far, most studies focused on adults but seldom children, which resulted in the limited data in our country. Here, we present a single-center study of PLA and analysis clinical characteristics and pathogens in pediatric population.

\section{Methods}


In this retrospective study, we reviewed data from children (younger than 18 years old) who admitted to Beijing Children's hospital (National Center for children's Health, China; A 970-bed tertiary pediatric hospital) and diagnosed PLA from January 2017 to June 2019. All of the patients met with at least one of the following inclusion criteria ${ }^{[11]}$ :(1) Presence of abscess in the liver on imaging examinations; (2) Confirmed by aspiration for bacteriology studies. We collected the information of the risk factors, clinical presentations, laboratory tests including pathogen results, radiological findings, management and outcomes of PLA patients.

\section{Statistical analysis}

Mean and standard deviations (SDs) are shown when distributions were confirmed normal; median and interquartile ranges (IQRs) are reported otherwise. The categorical variables were compared using the Chisquare test or Fischer's exact test, as appropriate. Continuous variables within two groups were compared using the independent t-test for parametric data and the Mann-Whitney $U$ test for non-parametric data. $P$ values $<0.05$ were considered statistically significant. All of the statistical analyses were conducted using SPSS 23.0 (SPSS Inc., USA)

\section{Results}

\section{Baseline characteristics of the patients}

Fifteen children were diagnosed as PLA from January 2017 to June 2019, including 8 males (53.3\%) and 7 females $(46.7 \%$ ), the median age of onset was 3.2 (range $0.17,5.6$ )years, the minimum was 17 -day-old and the median diagnostic time was 9Dinterquartile range 5, 22】days. Five (33.3\%) patients had been treated in intensive care unit (ICU). The clinical characteristics of patients with PLA are concluded in Table 1.

\section{Predisposing factors}

Among 4 out of 15 patients (26.7\%) who had underlying diseases, one was juvenile idiopathic arthritis with operation of abdomen and another one had acute lymphoblastic leukemia, both of whom had been received a long-time treatment of glucocorticoid and immunosuppressive drugs. And one patient was DM, another one was primary immunodeficiency.

\section{Clinical presentations}

Thirteen patients( $86.7 \%)$ had fever, nine $(60 \%)$ of whose temperature were over $39^{\circ} \mathrm{C}$. Four patients $(26.7 \%)$ suffered from abdominal pain and two patients $(13.3 \%)$ presented with cough. Metastasis were observed in 7 patients (46.6\%) with multiple organ systems involved (Fig. 1). Four patients (26.7\%) had splenic abscess, two patients (13.3\%) had intra-abdominal infections and other extrahepatic complications such as pneumonia, meningitis, skin infection, urinary tract infection, endocarditis, infection of hip joint and spine were also observed. 


\section{Laboratory data}

\section{Blood tests}

C-reactive protein (CRP) level was elevated in 13 patients (86.7\%), increased procalcitonin (PCT) level was observed in 11 patients (84.6\%) and leukocytosis was showed in 12 patients (80.0\%). Five patients (33.3\%) had elevated level of alanine transaminase.

\section{Pathogen findings}

Aspirations were done in 5 patients (33.3\%) and one of them was confirmed the infection of $\mathrm{K}$. pneumonia through pus culture, moreover another two were identified Streptococcus intermedius (unique reads 3 ) and Bartonella henselae(unique reads 92 ) separately by the pus of metagenomic nextgeneration sequencing (mNGS). Thirteen patients were taken blood culture, Staphylococcus aureus (Methicillin-resistant staphylococcus) was isolated from one patient and Pseudomonas aeruginosa (carbapenemase positive) was isolated from another patient. The rest had negative results of blood and pus culture.

\section{Imaging findings}

All patients performed ultrasound and nearly one half (46.7\%) performed the contrast-enhanced computed tomography (CT) scan simultaneously. The detail features were shown in Table 1. Six patients $(40.0 \%)$ had single abscess, the rest was multiple. Eleven out of 15 patients $(73.3 \%)$ had unilateral involvement and 8 patients (53.3\%) occupied right lobe. Thirteen patients presented with low hypoechoic lesions in ultrasound, two patients were normal. Seven patients (46.7\%) who underwent contrastenhanced CT scan showed lesions of low density with edge intensified (Fig. 2).

\section{Therapy and outcomes}

All patients received intravenous antimicrobial therapy, nine patients $(60.0 \%)$ with the treatment of single agent initially (Fig. 3), three of which changed antimicrobial treatment after obtaining the result of pathogen. Among one patient received single agent of meropenem and then combined linezolid at the time detected Streptococcus intermedius by mNGS. And one patient who was proved Bartonella henselae changed from cefatriaxone to rifampicin together with azithromycin. Initial treatment of one patient was cefoperazone/sulbactam and pathogen of liver pus culture was negative, but K.pneumonia was isolated from pus culture of intra-abdominal and then changed meropenem companied with vancomycin. Finally eleven patients $(73.3 \%)$ took a favorable turn and were confirmed to be cured through imaging findings during follow-up time. Four patients (26.7\%) discharged without treatment and then dead. Compared to the survival group and the death group, the patients in death group were more likely to have multiple organ involved $(100$ vs. $27 \%, P<0.05)$ and bilateral lobe occupied $(P<0.05)$.

\section{Discussion}


HA is invaded by microbes via bile ducts, blood stream (hepatic artery and portal vein) or by contiguous spread $^{[11,12]}$ and PLA is accused of the majority causes of $\mathrm{HA}^{[1]}$. Numerous studies show the risk factors which predispose patients to PLA, including immune-compromised state (immunodeficiency, chemotherapy and etc.), DM and so on ${ }^{[13-15]}$. In our research, two patients took glucocorticoid and immunosuppressive drugs and one patient had primary immunodeficiency, which were belonged to a state of immune-compromising so that it was susceptible to form abscess. Also, another patient was considered severe combined immunodeficiency due to the significant decreased in serum immunoglobulin and $T$ cell subsets, however it was unable to confirm the diagnosis that his parents refused to do genetic test. Furthermore one patient had DM which was noted as a condition that immune system was wakened ${ }^{[15]}$, thus getting infected easily.

Almost all patients (86.7\%) had fever and a fair of patients $(26.7 \%)$ presented abdominal pain, which could be the most common clinical features and basis to make the diagnosis of PLA ${ }^{[9]}$. Meanwhile several patients had cough due to the stimulation of diaphragm through liver abscess nearby and according to some studies that patients complain of non-specific symptoms such as fatigue, anemia and weight loss ${ }^{[16]}$. Which is easy to misdiagnose during clinical assessment and requires concerning on PLA when treat these patients, especially for immunosuppressed populations.

At the initial state of disease, most patients(86.7\%) had elevated level of CRP, eleven patients(84.6\%) had increased level of PCT and twelve patients $(80 \%)$ presented with leukocytosis, all of these indicated the infection and especially for bacterium, which was proved in various studies of PLA ${ }^{[1,16]}$. More than half (53.3\%) had abscess in the right lobe, could be correlated with blood flow direction and volume ${ }^{[1]}$. Two patients with small multiple abscesses were detected by CT scan, but undetected by ultrasound. The sensitivity achieved $100 \%$ when ultrasound and contrast-enhanced CT scan were combined use. Actually ultrasound was more widely used in clinical work due to universality of technology, ease of operation and higher cost performance, even as a tool to perform ultrasound-guild aspiration. It has been reported contrast-enhanced CT scan was done in PLA patients ${ }^{[16-18]}$, but owing to the potential risk of liver or renal function impaired and the consideration of economic burden, ultrasound became one of the main imaging examination method to diagnose PLA.

In our study, K.pneumonia was isolated from a patient with DM through pus culture. Another patient whose pus culture of liver abscess was negative, whereas K.pneumonia was isolated through pus culture of intra-abnormal abscess, which could be suspected that the pathogen of PLA in this patient was K.pneumonia probably. In the past few decades, S.aureus is the most common pathogen isolated from PLA in children all over the world ${ }^{[8,9]}$. Whereas in recent years, with the depth understanding of disease and improvement of laboratory technology, the pathogen of PLA have changed. In a meta-analysis of pathogen distribution with PLA in China ${ }^{\left[{ }^{[8]}\right.}$, Klebsiella spp was the most common pathogen and mostly occurred in DM. K.pneumonia strain is mostly K1 or K2 serotype. Neutrophil phagocytosis and chemotaxis may be impaired due to the poorly controlled of blood glucose ${ }^{[12,15,19]}$, leading to infected with K.pneumonia easily. 
Culture-based techniques are the golden standard, and varies studies advanced more pathogens can be

isolated through pus culture ${ }^{[18,20,21]}$. In our study the positive rate of blood or pus culture was not very high, it could be attribute to sample collection after prior antibiotic treatment of pre-hospitalization. Meanwhile, it is of difficulty to cultivate in restricting conditions and takes a long time to get results which are regarded as limitation of culture and isolation of pathogen.

It is worth noting that in our study patients infected with Streptococcus intermedius(unique reads 3 ) and Bartonella henselae(unique reads 92) were detected by mNGS, a new detection technology in recent years and presenting with promising result in the field of pathogen diagnosis ${ }^{[22]}$. A study in our center about evaluation of mNGS for the pathogenic diagnosis showed the ability to identify pathogens from abscess and less affected by prior antibiotic ${ }^{[23]}$. Applying this technology in clinical practice to gain more experiences is our direction in the future.

All patients received antibiotics on account of susceptibility and experience, the monotherapy with meropenem or third-generation of cephalosporin was the main regimen in our study (53.3\%), which conformed to the pathogen distribution of PLA in our country ${ }^{[8]}$. In one patient, the liver abscess was enlarged after receiving antibiotics only and then finished aspiration. It is recommend ${ }^{[24]}$ that all patients should be given antibiotics and evaluated therapy response in 48 hours, drainage is necessary to be done when no response, incomplete response or abscess enlarged. If US showed the abscess rupture or impending rupture, large with the location of left lobe, requiring intervention because of associated abdominal lesion, urgent drainage was demanded. In our study, the rate of complications was $46.7 \%, 57 \%$ (4/7) of which were dead. A research indicated about $15.7 \%$ of HA patients develop complications, which can be accused of most death ${ }^{[19]}$.

This study has some limitations. It was a single center, hospital-based retrospective design, which introduces the possibility of unrecognized biases. Abscess biopsy was done in seldom patients and due to the usage of antibiotics before admitted to our hospital, the positive rate of pathogen was not very high. Further multicenter studies are necessary to enrich the generalizability of PLA in children.

\section{Conclusions}

PLA often occurs in patients with underlying disease. Multiple organs involved and bilateral lobe occupied may lead to poor prognosis. mNGS can be a new method to detect pathogen.

\section{Abbreviations}

\section{PLA}

Pyogenic liver abscess; mNGS:metagenomic next-generation sequencing; HA:hepatic abscess; S.aureus:Staphylococcus aureus; E. Coli:Escherichia coli ; K.pneumonia:Klebsiella pneumonia; DM:Diabetic mellitus; SDs:mean and standard deviations; IQRs:interquartile ranges; PID:primary immunodeficiency disease; SCID:Severe Combined Immunodeficiency Disease; ALL:acute lymphatic 
leukemia; CNS:central nervous system; JIA:juvenile idiopathic arthritis; TZP, piperacillin/tazobactam; CRO, cefatriaxone; SCF, cefoperazone/sulbactam; MEM, meropenem; VA, vancomycin; RD, rifampicin; AZM, azithromycin; MTZ, metronidazole; LZD, linezolid; SAM, ampicillin/sulbactam; ZOX, ceftizoxime; ICU:intensive care unit; CRP:C-reactive protein; PCT:procalcitonin; CT:computed tomography; WBC:white blood cell; ALT:transaminase.

\section{Declarations}

\section{Acknowledgments}

We would like to thank the Medical Records and Statistics Room, Beijing Children's Hospital, which provided hospital discharge data.

\section{Funding}

This work was supported by Beijing Hospital Authority "Dengfeng" Talent Training Plan (DFL 20181201). The funders had no role in study design, data collection and analysis, or preparation of the manuscript.

\section{Availability of data and materials}

The datasets during and/or analyzed during the current study are available from the corresponding author on reasonable request.

\section{Authors' contributions}

All of the authors had access to the full dataset (including the statistical reports and tables) and take responsibility for the integrity of the data and the accuracy of the data analysis. GL, LYG and YX conceived and designed the study. YX, LYG, LLL, BL, WYF, HLH, BH, XG, TMC, SYL, SYQ, MYH and HYZ were involved in the case and sample collection, designed the analysis and interpreted the data. $Y X$ wrote the first draft of the paper. YX, LYG and GL reviewed and approved the final report. All authors have read and approved the final manuscript.

\section{Competing interests}

The authors declare that they have no competing interests. 


\section{Consent for publication}

Not applicable.

\section{Ethics approval and consent to participate}

This study was reviewed and approved by the Ethics Committee of Beijing Children's Hospital Affiliated to Capital Medical University (2019-k-374). Because this is a retrospectively study, we obtained the data of patients from the Medical Records and Statistics Room and we analyzed the data anonymously; thus, informed consent was not required.

\section{Author details}

${ }^{1}$ Key Laboratory of Major Diseases in Children, Ministry of Education, Department of Infectious Diseases, Beijing Children's Hospital, Capital Medical University, National Center for Children's Health, No. 56 Nan Lishi Road, Beijing 100045, China. ${ }^{2}$ Intensive Care Unit, Beijing Children's Hospital, Capital Medical University, National Center for Children's Health, No. 56 Nan Lishi Road, Beijing 100045, China. ${ }^{3}$ Neonatal Center, Beijing Children's Hospital, Capital Medical University, National Center for Children's Health, No. 56 Nan Lishi Road, Beijing 100045, China. ${ }^{4}$ National Key Discipline of Paediatrics, Haematology and Oncology Center, Beijing Children's Hospital, Capital Medical University, National Center for Children's Health, No. 56 Nan Lishi Road, Beijing 100045, China.

\section{References}

1. Mishra K, Basu S , Roychoudhury S, et al. Liver abscess in children: an overview[J]. World Journal of Pediatrics, 2010, 6(3):210-216.

2. Huang C J , Pitt H A , Lipsett P A, et al. Pyogenic hepatic abscess. Changing trends over 42 years.[J]. Annals of Surgery, 1996, 223(5):600.

3. Ferreira M A B , Pereira F E L, Musso C , et al. Pyogenic liver abscess in children: Some observations in the Espirito Santo State, Brazil[J]. Arquivos de Gastroenterologia, 1997, 34(1):49-54.

4. Alok Kumar, S Srinivasan, AK Sharma. Pyogenic liver abscess in children - South Indian experiences[J]. Journal of Pediatric Surgery, 1998, 33(3):417-421.

5. Pineiro-Carrero V M , Andres J M . Morbidity and mortality in children with pyogenic liver abscess[J]. American journal of diseases of children (1960), 1990, 143(12):1424-1427.

6. Kong M S,Lin J N,Pyogenic liver abscess in children.[J] .J. Formos. Med. Assoc., 1994, 93: 45-50. 
7. Chia-Chang Tsai, Jiin-Haur Chung, Sheung-Fat Ko, et al. Liver Abscess in Children: A Single Institutional Experience in Southern Taiwan[J]. Acta Paediatrica Taiwanica, 2003, 44(5):282-286.

8. Luo $M$, Yang $X X$, Tan $B$, et al. Distribution of common pathogens in patients with pyogenic liver abscess in China: a meta-analysis[J]. European Journal of Clinical Microbiology \& Infectious Diseases, 2016, 35(10):1557-1565.

9. Muorah M , Hinds R, Verma A, et al. Liver Abscesses in Children: A Single Center Experience in the Developed World[J]. Journal of Pediatric Gastroenterology and Nutrition, 2006, 42(2):201-206.

10. Mavilia M G , Molina M , Wu G Y . The Evolving Nature of Hepatic Abscess: A Review[J]. Journal of Clinical and Translational Hepatology, 2016, 4(2).

11. Lardière-Deguelte S,Ragot E,Amroun K et al. Hepatic abscess: Diagnosis and management.[J] .J Visc Surg, 2015, 152: 231-43.

12. Jun Jae Bum,Klebsiella pneumoniae Liver Abscess.[J] .Infect Chemother, 2018, 50: 210-218.

13. Lee K T , Wong S R, Sheen P C . Pyogenic Liver Abscess: An Audit of 10 Years' Experience and Analysis of Risk Factors / with Invited Commentary[J]. Digestive Surgery, 2002, 18(6):459-466.

14. Lublin M , Bartlett D L , Danforth D N, et al. Hepatic Abscess in Patients With Chronic Granulomatous Disease[J]. Annals of Surgery, 2002, 235(3):383-391.

15. Eltawansy S A, Merchant C , Atluri P, et al. Multi-Organ Failure Secondary to a Clostridium Perfringens Gaseous Liver Abscess following a Self-Limited Episode of Acute Gastroenteritis[J]. American Journal of Case Reports, 2015, 16:182-186.

16. Sharma M P , Kumar A . Liver abscess in children[J]. The Indian Journal of Pediatrics, 2006, 73(9):813-817.

17. Shin $S U$, Park C M , Lee $Y$, et al. Clinical and radiological features of invasive Klebsiella pneumoniae liver abscess syndrome[J]. Acta Radiologica, 2013, 54(5):557-563.

18. Hsu YL,Lin HC,Yen TY,et al. Pyogenic liver abscess among children in a medical center in Central Taiwan. [J].Journal of Microbiology Immunology and Infection 2015, 48(3):302-305.

19. Chen $\mathrm{CH}$, Wu S S , Chang H C , et al. Initial presentations and final outcomes of primary pyogenic liver abscess: a cross-sectional study[J]. BMC Gastroenterology, 2014, 14(1).

20. Fazili T , Sharngoe C , Endy $T$, et al. Klebsiella pneumoniae Liver Abscess: An Emerging Disease[J]. The American Journal of the Medical Sciences, 2016, 351(3):297-304.

21. Yu S C H , Ho S S M , Lau W Y, et al. Treatment of pyogenic liver abscess: Prospective randomized comparison of catheter drainage and needle aspiration[J]. Hepatology, 2004, 39(4):932-938.

22. Gong Liang, Huang Yao-Ting, Wong Chee-Hong, et al. Culture-independent analysis of liver abscess using nanopore sequencing[J]. Plos One, 13(1):e0190853-.

23. Hu H L , Guo L Y, Wu HL, et al. Evaluation of next-generation sequencing for the pathogenic diagnosis of children brain abscesses[J]. Journal of Infection, 2019.

24. Srivastava A, Yachha S K, Arora V , et al. Identification of high-risk group and therapeutic options in children with liver abscess[J]. European Journal of Pediatrics, 2012, 171(1):33-41. 


\section{Tables}

Due to technical limitations, tables are only available as a download in the supplemental files section

\section{Highlights}

Pyogenic liver abscess often occurs in patients with underlying disease.

Multiple organs involved and bilateral lobe occupied may lead to poor prognosis.

Metagenomic next-generation sequencing could be a new method to make diagnosis.

\section{Figures}

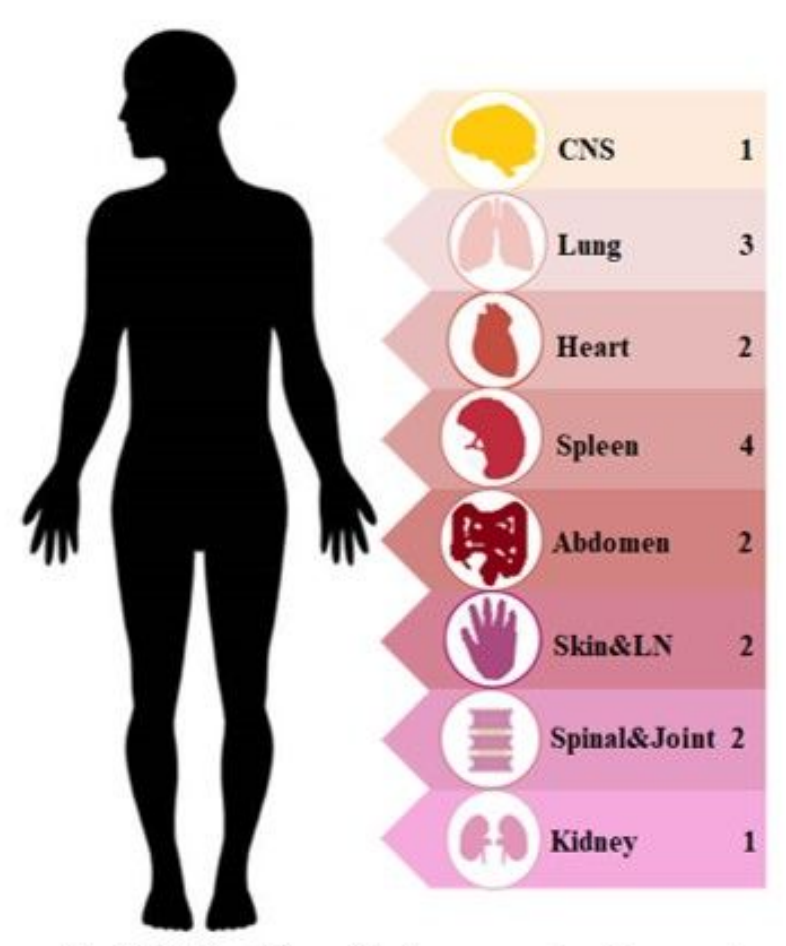

Fig.1 PLA with multiple organs involvement CNS, central nerves system; LN, lymph node; 


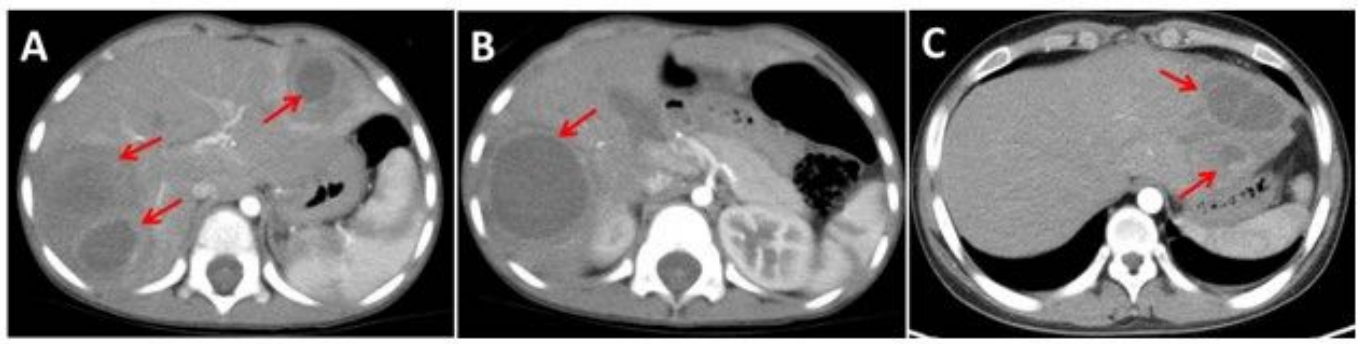

Fig. 2 Imaging findings of pediatric PLA cases. Contrast-enhanced CT scan showed lesions were low density, well-defined and mostly similar to the round with edge intensified. A Multiple liver abscesses with bilobar involved. B Single abscess in right lobe. C Multiple liver abscesses in left lobe.

\section{Figure 2}

Imaging findings of pediatric PLA cases. Contrast-enhanced CT scan showed lesions were low density, well-defined and mostly similar to the round with edge intensified. A Multiple liver abscesses with bilobar involved. B Single abscess in right lobe. C Multiple liver abscesses in left lobe. 


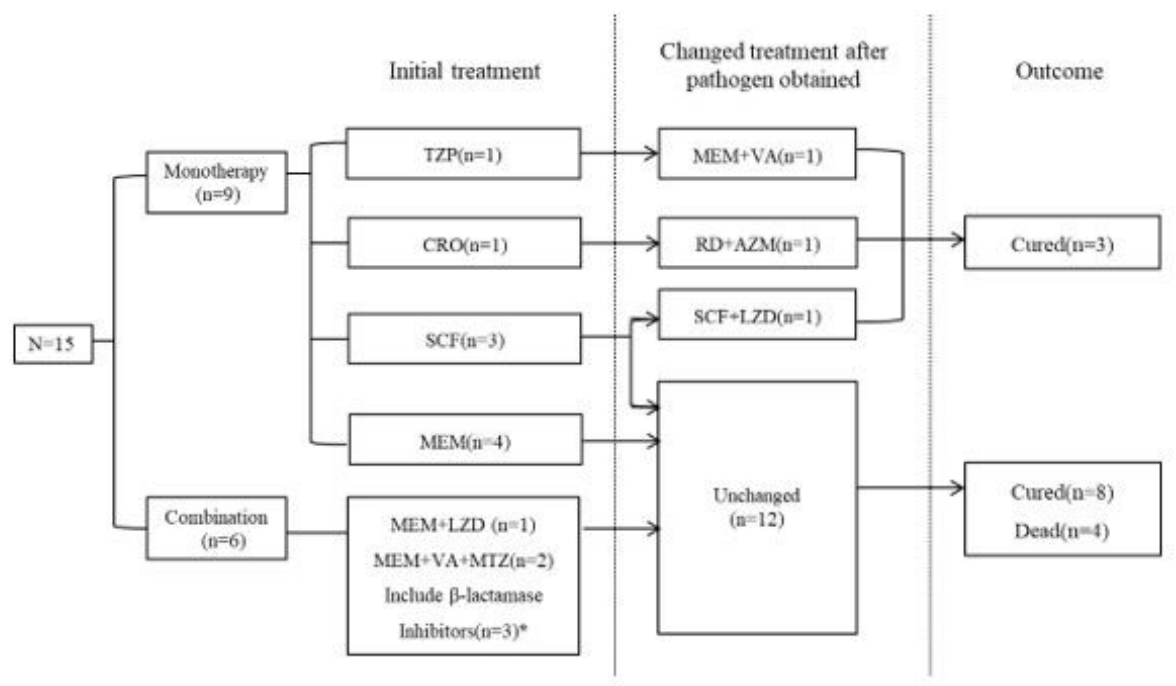

Fig.3Antibiotic treatment and outcome of patients with PLA

TZP, piperacillin tazobactam; CRO, cefatriaxone; SCF, cefoperazone/sulbactam; MEM, meropenem; VA, vancomycin; $\mathrm{RD}$, rifampicin; AZM, azithromycin; MTZ, metronidazole; LZD, linezolid; $\mathrm{SAM}$, ampicillin/sulbactam; ZOX, ceftizoxime; *TZP+LZD+RD; SAM+ZOX; SCF+MTZ

\section{Figure 3}

Antibiotic treatment and outcome of patients with PLA TZP, piperacillin/tazobactam; CRO, cefatriaxone; SCF, cefoperazone/sulbactam; MEM, meropenem; VA, vancomycin; RD, rifampicin; AZM, azithromycin; MTZ, metronidazole; LZD, linezolid; SAM, ampicillin/sulbactam; ZOX, ceftizoxime; *TZP+LZD+RD; $\mathrm{SAM}+\mathrm{ZOX} ; \mathrm{SCF}+\mathrm{MTZ}$

\section{Supplementary Files}

This is a list of supplementary files associated with this preprint. Click to download.

- Table.1ClinicalcharacteristicsofpatientswithPLA.docx

- Table.2Comparison.docx 